\title{
Decompressing manubriectomy under apneic oxygenation to release the median thoracic outlet compartment in Bechterew disease
}

\author{
Tetsuhiko Go, MD, Matthias Altmayer, MD, Matthias Richter, MD, and Paolo Macchiarini, MD, PhD, Hannover, Germany
}

$\mathrm{I}$ ndividuals with long-lasting ankylosing spondylitis (AS, Bechterew disease) are at increased risk for developing fractures as the fused spine often becomes osteoporotic. These bony protuberances may compress all elements lying in front of them and induce a soft-tissue inflammation to the extent that it causes irreversible dysphagia, especially at the level of the cervical spine. ${ }^{1} \mathrm{We}$ describe here the decompression of the entire visceral mass of the median thoracic aperture by a simple manubriectomy in an AS patient with refractory dyspnea and dysphagia.

\section{Clinical Summary}

A 62-year-old man with a 15-year history of AS was referred to our department for treatment of severe dyspnea and solid dysphagia of unknown cause. His medical history was significant for chronic alcohol abuse. Recently, he developed solid dysphagia and became increasingly dyspneic to the extent that he had his first episode of asphyxia, requiring an emergency nasotracheal intubation and mechanical ventilation. Initial fibroscopy revealed a massive laryngeal edema and a suboccluded subglottic region; plain cervical radiology was negative and computed tomography (CT) showed a massive prevertebral soft-tissue swelling. Following the acute episode, the patient was weaned and an extubation was attempted twice over a period of 1 month. Both attempts failed because of the persistence of the cervical soft-tissue swelling despite aggressive anti-inflammatory and steroid therapy. Because of that, a nasotracheal tube was used; the tip was placed below the edematous upper airway and the patient was weaned and allowed to breathe spontaneously. He was referred to us 2 weeks thereafter with the intent to perform a definitive mediastinal tracheostomy.

Examination revealed a global restriction of spinal movements in keeping with long-standing AS without neurological deficits (with the exception of motor disturbance of his right fingers). Of note was that the chin to manubrium sterni angle was less than $20^{\circ}$. Close questioning revealed only that following a neck hyperextension 2 years ago, he had transient neck pain, which disappeared thereafter. Because of this and to rule that the swelling was an

From the Department of Thoracic and Vascular Surgery, Heidehaus Hospital, Hannover Medical School, Hannover, Germany.

Received for publication June 10, 2002; revisions requested July 8, 2002; revisions received July 10, 2002; accepted for publication July 18, 2002.

Address for reprints: Paolo Macchiarini, MD, PhD, Department of Thoracic and Vascular Surgery, Heidehaus Hospital (Hannover Medical School), Am Leineufer 70, 30419, Hannover, Germany (E-mail: pmacchiarini@ compuserve.com).

J Thorac Cardiovasc Surg 2003;126:867-9

Copyright $(2003$ by The American Association for Thoracic Surgery $0022-5223 / 2003 \$ 30.00+0$

doi:10.1016/S0022-5223(03)00394-5 artifact due to an inappropriately placed endotracheal tube, ${ }^{2}$ a spiral 3-mm thin slice CT in sagittal view was made, which showed the presence of a fracture dislocation at the C6-7 intervertebral disc space, with forward slip of C-6 on C-7, and a massive swelling of all visceral mass lying between the $\mathrm{C} 4-7$ prevertebral plane dorsally and manubrium sterni ventrally (Figure 1). Rigid bronchoscopy revealed remarkable edematous changes of the entire laryngeal and upper cervical tracheal mucosa to the extent that it permitted only the risky passage of the pediatric bronchoscope; the distal trachea and bronchial system were normal. Several biopsies of the upper airway ruled out a malignant disease and showed only inflamed and edematous tissue. The nasogastric tube was removed but because of its intrinsic risks, an esophagoscopy was not made.

Referring to the spiral CT and his medical history, we speculated that all the visceral mass lying between the prevertebral plane and the manubrium sterni had been gradually compressed since his cervical fracture occurred, resulting in its refractory inflammation and swelling. Despite the generalized spinal rigidity and exaggerated thoracic kyphosis, we felt that by doing a simple manubriectomy and placing a temporary mediastinal tracheotomy, all the hypopharynx and laryngotracheal masses would be allowed to slip ventrally and decompress, which would reduce or eliminate swelling. On the other hand, a surgical stabilization of the spinal fracture was considered unnecessary by the spine surgeons.

The operation was performed under general anesthesia through the original nasotracheal tube using continuous infusion technique with short-acting muscle relaxants, rapidly metabolized intravenous anesthetic agents, and short-acting opioids. The patient was placed in the supine position, and maximal care was given to maintain the original position of the neck to avoid a hyperextension of his head. A transversal myocutaneous incision, similar to that used for a mediastinal tracheostomy in cervical exenteration, was made while the first assistant held the patient's jaw to maintain visualization of the operative field. The sternohyoid muscles were divided in the midline, and the thyroid isthmus was divided and ligated to expose the anterior surface of the cervical trachea. Inspection showed that the laryngotracheal axis had sunk vertically into the retrosternal space and, due to his kyphosis, the upper border of the manubrium sterni had compressed the cricoid and upper 2 tracheal rings. After exposing the manubrium sterni and clavicle heads, a standard manubriectomy was performed, preserving the sternoclavicular junctions bilaterally (Figure 2). This simple maneuver was sufficient to elevate spontaneously the laryngotracheal axis ventrally for at least $3 \mathrm{~cm}$. After the removal of the nasotracheal tube, the larynx was controlled endoscopically from above, and a section of the trachea was made between the second and third cartilage rings to carefully rule out significant postintubation fibrotic stenosis or other diseases.

Because of the very limited surgical exposure, systemic oxygenation was assured by combining a 14-minute preoxygenation 


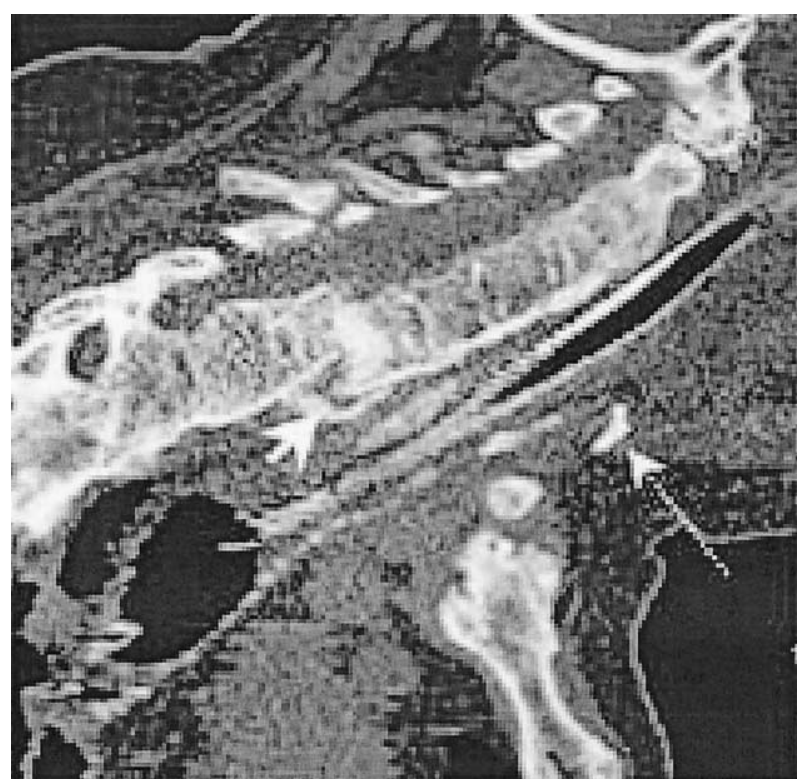

Figure 1. Preoperative spiral 3-mm CT (lateral view) showing the fracture dislocation at the C6-7 intervertebral disc space, with forward slip of C-6 on C-7 (solid rule with arrowhead) and the endotracheal tube that maintained patency of the upper airway. Of note also is the narrowed space below the hyoid bone where conventional tracheostomy is unfeasible (dotted rule with arrowhead).

phase (with $100 \% \mathrm{FIO}_{2}$ ) with a 33-minute apneic oxygenation phase using a pediatric nasogastric tube placed above the carina and delivering $10 \mathrm{~L}$ of oxygen per minute (Figure 3 ). This allowed visual inspection, mucosal sampling, and estimation of the extent of the disease.

No fibrotic lesions were observed, only severe edematous mucosal changes reaching the second tracheal ring, and frozen sections were negative for primary or secondary airway diseases.

We postulated that by associating the manubriectomy to a temporary tracheostomy placed well below the previously made tracheal section, all the swelling would restore. Therefore, the tracheal section was closed with absorbable interrupted sutures, and a new section was made between the fifth and sixth tracheal rings to place a conventional tracheostomy tube. A viable muscle was interposed between the tracheotomy tube and the brachiocephalic artery; a drainage tube was left beside the trachea and the wound was closed.

The postoperative course was uneventful. Dysphagia completely disappeared by day 7 , and the dsypnea improved to such an extent that the tracheostomy tube was removed by day 17 . The patient was breathing normally without impairment by day 21 . By then, control fibroscopy was normal and a cervical CT showed a patent larynx and upper trachea (Figure 4).

\section{Comments}

The necessity of handling the spine with extreme caution during all medical procedures in patients with AS cannot be overstressed due to the osteporotic status of the fused spine and the underlying risks

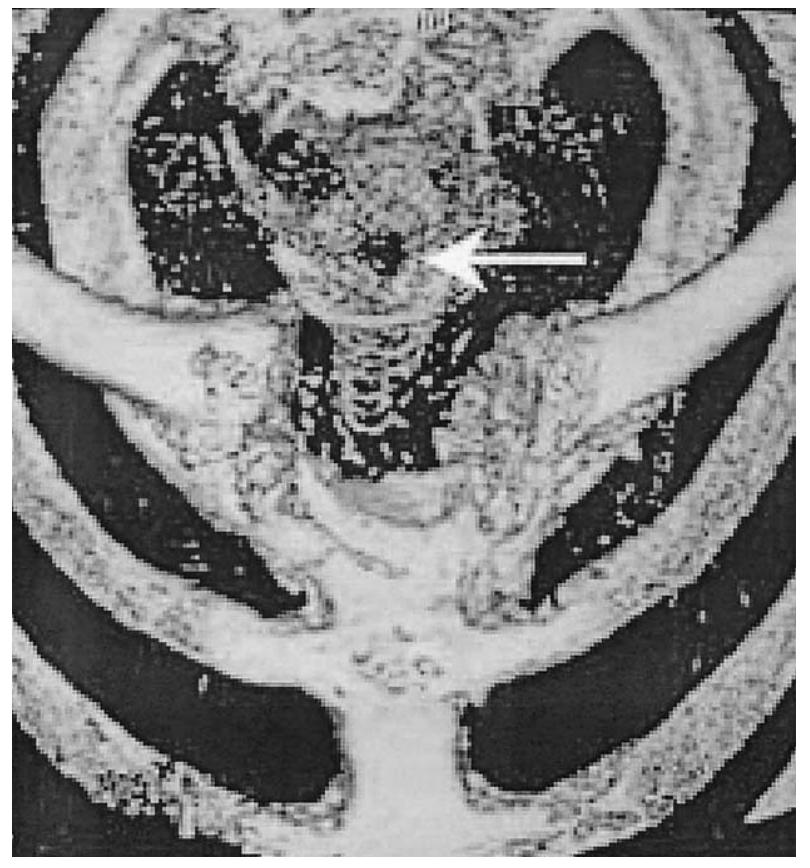

Figure 2. Postoperative CT (frontal view) showing the enlarged space between the spine and the created fenestration that was obtained following the manubriectomy, where the cervical airway is patent (arrow).

of irreversible neurological damages. Generalized spinal rigidity and exaggerated thoracic kyphosis may hinder the anterior exposure in these patients; injury to the upper digestive tract or airway is the result of bony protuberances, and inappropriate management may result in excessive neurological injury and mortality. ${ }^{1}$ In our patient, the soft-tissue inflammation of the entire visceral mass lying in the median thoracic aperture was the result of a "forceps" effect induced by the exaggerated thoracic kyphosis and subclinical vertebral luxation dorsally and the rigid bony plane of the manubrium sterni ventrally.

By doing a simple manubriectomy and placing a temporary mediastinal tracheostomy, we were able to decompress the entire structure and the organs between the spine and sternum and allow the patient to completely recover symptomatically. The major point of interest of this report is the surgical management. Manubriectomy is usually performed to resect primary or secondary tumor processes of structures or organs lying in the median cervical region or for primary bony lesions. In the present case, it was successfully performed to relieve the airway and avoid a permanent mediastinal tracheostomy along with its related inconveniences. By combining this simple procedure with a temporary tracheotomy, the mechanical resistance of the manubrium sterni was removed, and the free space created, along with the decompressing tracheotomy, allowed the steroid-refractory swelling of the entire visceral mass of the median thorax aperture to subside. Because the upper esophagus and airway regained their normal anatomical position and physiology, the patient could return to normal swallowing and breathing functions within 20 days postoperatively. 


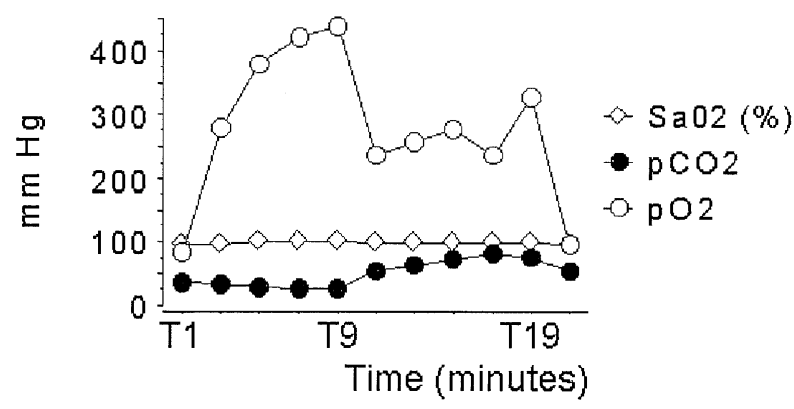

Figure 3. Intraoperative trend of the arterial blood gases measured at intervals of 2 (preoxygenation phase) and 3 (apneic oxygenation phase) minutes. T1 corresponds to the baseline value; T2 to T8 is the preoxygenation phase (patient is hyperventilated with an inspired oxygen concentration of 100\%); T9 to T19 is the apneic oxygenation phase (insufflation of $10 \mathrm{~L} / \mathrm{min}$ through a catheter above the carina, without ventilation); and T19 to T22 is the postapneic phase (patient is volume-controlled reventilated with an inspired oxygen concentration of $30 \%$ ). $\mathrm{SaO}_{2}$, oxygen saturation; $\mathrm{pCO}_{2}$, partial carbon dioxide; $\mathrm{pO}_{2}$, partial oxygen.

Given the anatomical modification of the neck, we were concerned with the feasibility of placing the traditional cross-field tube ventilation system ${ }^{3}$ into the distal trachea during the opening of the trachea. Using the most recent contributions to the area of apnea oxygenation ${ }^{4}$ and the lessons from others, ${ }^{5,6}$ we used the physiological concept of the intrapulmonary oxygen store (eg, the preoxygenation and apneic oxygenation) for the first time in tracheal surgery. Before sectioning the airway, the patient was preoxygenated and hyperventilated with $100 \%$ oxygen only so that the 2500 to $3000 \mathrm{~mL}$ volume of his functional residual capacity was almost completely denitrogenated and any nitrogen entrainment ceased. As the normal oxygen consumption is 200 to $250 \mathrm{~mL}$ per minute, the 2500 - to $3000-\mathrm{mL}$ intrapulmonary oxygen store provides adequate oxygenation for 10 to 12 minutes of total apnea. Furthermore, after the opening of the airway, a pediatric catheter was placed under visualization above the carina, and by simply delivering a small flow (10 to $15 \mathrm{~L}$ of oxygen per minute) under minimal breathing pressure ( 0 to 2 psi) to both lungs, the patient could "breathe" despite ongoing total apnea; the patient could even survive apneic periods of 50 minutes without any ill effects (P.M., personal communication). In contrast to McClish and others, ${ }^{6}$ we do not ventilate the lungs and we use a low-pressure (0 to 2 psi) and small-flow (10 to $15 \mathrm{~L}$ of oxygen per minute) delivering system. This represents, in our opinion, a more physiological method of ventilation while keeping the significant advantages of the catheter technique (eg, improved surgical exposure, minimal intraoperative intrusion of the anesthetic apparatus, and facilitated reconstruction).

In conclusion, a permanent tracheostomy was avoided by performing a simple manubriectomy and placing a temporary tracheostomy in a patient with AS. This simple maneuver allowed complete symptomatic relief. The preoxygenation and apneic ox-

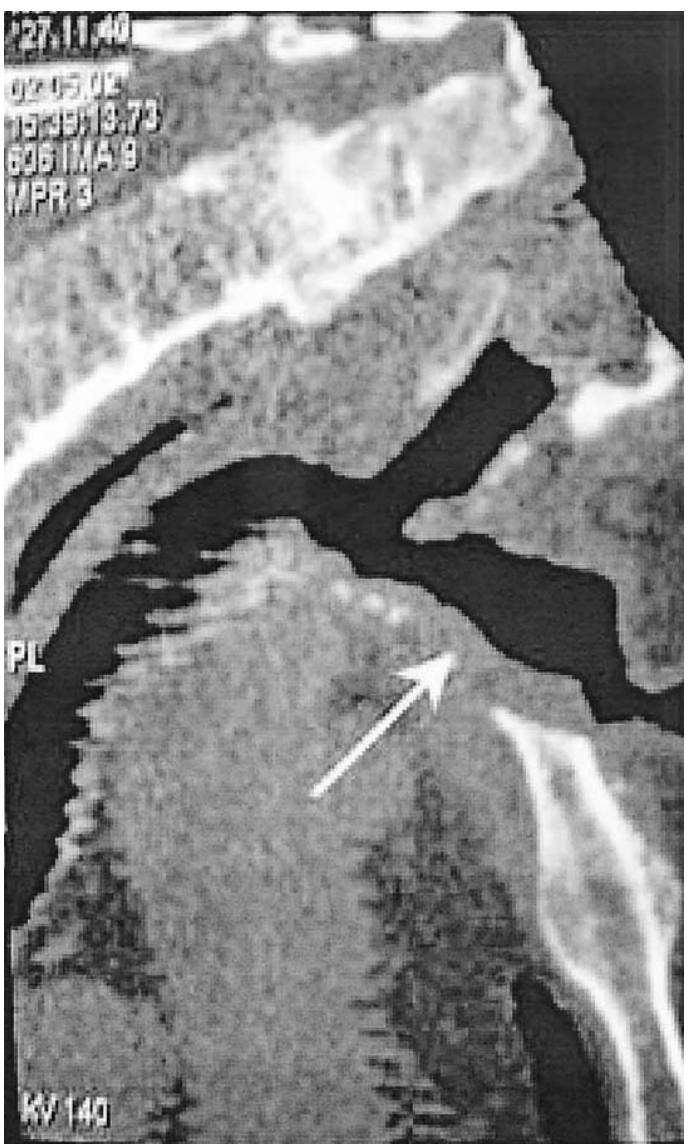

Figure 4. Postoperative spiral 3-mm CT (lateral view) showing a patent upper airway and the route of the mediastinal tracheostomy (arrow).

ygenation with the low-pressure (0 to 2 psi) and small-flow (10 to $15 \mathrm{~L}$ of oxygen per minute) catheter technique represent a valid anesthetic alternative to the cross-field intubation technique for complex tracheal operation where surgical exposure should be maximal and reconstruction uninterrupted.

\section{References}

1. Murray GC, Persellin RH. Cervical fracture complicating ankylosing spondilytis: a report of eight cases and review of the literature. Am J Med. 1991;70:1033-41.

2. Fox BM, Harries SR, Defriend DE, Hughes PM. Pre-cervical soft tissues: a cautionary note. Eur J Emerg Med. 2001;8:151-2.

3. Geffin B, Bland J, Grillo H. Anesthetic management of tracheal resection and reconstruction. Anesth Analg. 1969;48:884-94.

4. Zander R, Mertzlufft F. Clinical use of oxygen stores: pre-oxygenation and apneic oxygenation. Adv Exp Med Biol. 1992;317:413-20.

5. Frumin JM, Epstein RM, Cohen C. Apneic oxygenation in man. Anesthesiology. 1959;20:789-98.

6. McClish A, Deslauriers J, Beaulieu M, et al. High-flow ventilation during major tracheobronchial reconstruction. $J$ Thorac Cardiovasc Surg. 1985;89:508-12. 